\title{
Onset of chronic myeloid leukemia with complex karyotype in a pregnant patient: case report and revision of literature
}

This article was published in the following Dove Press journal:

Therapeutics and Clinical Risk Management

27 June 2017

Number of times this article has been viewed

\author{
Nicola Sgherza' \\ Elisabetta Abruzzese ${ }^{2}$ \\ Gianni Perla' \\ Maria Marta Minervini ${ }^{\prime}$ \\ Vincenzo Chiello' \\ Natale Sciannamè ${ }^{3}$ \\ Nicola Cascavilla' \\ 'Hematology, IRCCS "Casa Sollievo \\ della Sofferenza", San Giovanni \\ Rotondo (FG), ${ }^{2}$ Hematology, \\ Sant'Eugenio Hospital, Roma, \\ ${ }^{3}$ Gynecology and Obstetrics, IRCCS \\ "Casa Sollievo della Sofferenza", \\ San Giovanni Rotondo (FG), Italy
}

\begin{abstract}
Approximately $10 \%-12 \%$ of patients in chronic-phase chronic myeloid leukemia (CP-CML) have additional chromosomal aberrations at diagnosis; moreover, CML occurs in up to $10 \%$ of pregnancy-associated leukemias, with an annual incidence of 1 per 100,000 pregnancies. In this report we describe the case of a 36-year-old female with CP-CML diagnosed in the 18th week of pregnancy and with a new complex variant translocation $t(4 ; 9 ; 22 ; 21)(q 24 ; q 34 ; q 11 ; q 22)$ and an additional chromosomal aberration $\mathrm{t}(1 ; 20)(\mathrm{p} 36 ; \mathrm{p} 11)$. In consideration of her pregnancy, the patient strictly monitored her blood cell count without any specific treatment. At 32 weeks of pregnancy, the patient delivered via cesarean section a healthy baby girl. After 10 days from childbirth, dasatinib was started at a standard dosage of $100 \mathrm{mg}$ /day and 3 months later complete cytogenetic response and major molecular response were obtained, with the achievement of an optimal response according to European Leukemia Net recommendations and showing efficacy of this tyrosine kinase inhibitor (TKI) in the presence of a complex karyotype.
\end{abstract}

Keywords: chronic myeloid leukemia, complex karyotype, pregnancy, additional chromosomal aberration, variant translocation, tyrosine kinase inhibitor

\section{Introduction}

Chronic myeloid leukemia (CML) is a myeloproliferative neoplasm characterized by the presence of the Philadelphia chromosome $(\mathrm{Ph})$ resulting from the reciprocal translocation $\mathrm{t}(9 ; 22)(\mathrm{q} 34 ; \mathrm{q} 11) .{ }^{1}$ Approximately $10 \%-12 \%$ of patients in chronic-phase CML (CP-CML) present with additional chromosomal aberrations (ACAs) at diagnosis. ${ }^{2}$ Recently different researchers have studied this subgroup, including European Leukemia Net $(E L N)^{3}$ and have classified CML ACAs into "major" and "minor" route changes. ${ }^{4}$ The major route ACAs are the most common chromosomal abnormalities ( $>10 \%$ of cases with ACAs) and include trisomy 8, an extra Ph $(1 \operatorname{der}(22) \mathrm{t}(9 ; 22)$ $(\mathrm{q} 34 ; \mathrm{q} 11))$, isochromosome 17(i(17)(q10)), trisomy 19 and $\operatorname{ider}(22)(\mathrm{q} 10) \mathrm{t}(9 ; 22)$ (q34;q11). Other less common ACAs belong to minor route ACAs. Moreover, microdeletions can be present too and the occurrence of genomic microdeletions proximally to ABL1 or distally to BCR have been reported in CML cases with variant translocations with a greater frequency $(30 \%-40 \%)$ than in cases with classic $\mathrm{t}(9 ; 22)(10 \%-18 \%){ }^{5}$ When the chromosome changes are submicroscopic, the translocation can be masked and revealed only by fluorescence in situ hybridization (FISH) or by molecular analysis. ${ }^{6}$ Chromosome 9 deletions and variant translocations have no value for prognosis ${ }^{2,7,8}$ whereas ACA/Ph1 have been reported to have an adverse prognostic value, particularly in the case of the major route abnormalities, including, as described previously, trisomy 8 , an extra $\mathrm{Ph}(1 \operatorname{der}(22) \mathrm{t}(9 ; 22)(\mathrm{q} 34 ; \mathrm{q} 11))$, isochromosome $17(\mathrm{i}(17)(\mathrm{q} 10))$, hereby accept the Terms. Non-commercial uses of the work are permitted without any further permission from Dove Medical Press Limited, provided the work is properly attributed. For permission for commercial use of this work, please see paragraphs 4.2 and 5 of our Terms (https://www.dovepress.com/terms.php). 
trisomy 19, and ider(22)(q10)t(9;22)(q34;q11)., ${ }^{2,9}$ Major route $\mathrm{ACA} / \mathrm{Ph} 1$ at diagnosis do not mandate different initial treatments but represent a warning: this implies that the characteristics of the disease and the response to treatment require more frequent monitoring to permit timely changes in therapy in case of treatment failure. ${ }^{3}$ Major route ACA/ $\mathrm{Ph} 1$ developing during treatment were confirmed to be a signal of acceleration. ${ }^{10-12} \mathrm{CML}$ occurs in up to $10 \%$ of pregnancy-associated leukemias, with an annual incidence of 1 per 100,000 pregnancies. ${ }^{13}$ In this report we describe the case of a 36-year-old female diagnosed in her 18th week of pregnancy with CP-CML and presenting with a new complex variant translocation $\mathrm{t}(4 ; 9 ; 22 ; 21)(\mathrm{q} 24 ; \mathrm{q} 34 ; \mathrm{q} 11 ; \mathrm{q} 22)$ and an ACA t $(1 ; 20)(\mathrm{p} 36 ; \mathrm{p} 11)$. The rearrangement was analyzed by cytogenetic and FISH tests. To the best of our knowledge, this translocation has not been described in CML previously and specifically in a pregnant patient.

\section{Case report}

\section{Patient presentation}

A 36-year-old female, 18 weeks pregnant, presented in October 2015 with abnormal blood cell counts (white blood count $29.87 \times 10 * 9 / \mathrm{L}$, hemoglobin $11.5 \mathrm{~g} / \mathrm{dL}$ and platelets $592 \times 10 * 9 / \mathrm{L})$; evaluation of the peripheral blood smear revealed basophils $(2 \%)$, myelocytes $(7 \%)$, metamyelocytes $(13 \%)$, and blasts $(1 \%)$. Bone marrow aspiration was performed and its examination revealed a slightly hypercellular marrow with granulocytic hyperplasia. Cytogenetic analysis revealed the presence of a complex karyotype in all examined metaphases: 46 XX, t(1;20)(p36;p11), t(4;9;22;21) (q24;q34;q11;q22). Reverse transcriptase-polymerase chain reaction (RT-PCR) done on peripheral blood and bone marrow showed a b3a2 BCR/ABL fusion gene. The patient was diagnosed with $\mathrm{Ph}+\mathrm{CML}$ (low risk Sokal score). In consideration of her pregnancy and leukocyte and platelet values she was advised to strictly monitor her blood cell count, with no other treatment apart from a low dose of aspirin. At 32 weeks (January 2016), the patient delivered via cesarean section a healthy baby girl (weight 2,120 g, height $43 \mathrm{~cm}$; APGAR 9). At the time of childbirth, the hematological analysis revealed a white blood cell count of $73.51 \times 10 * 9 / \mathrm{L}$, a hemoglobin level of $10 \mathrm{~g} / \mathrm{dL}$, a platelet count of $494 \times 10 * 9 / \mathrm{L}$. After 10 days from childbirth, the patient started dasatinib at a standard dosage of $100 \mathrm{mg} /$ day. After 3 months on dasatinib the patient obtained complete cytogenetic response and major molecular response (MMR), achieving an optimal response according to the ELN recommendations.

\section{Cytogenetic analysis}

Cytogenetic analysis was performed on bone marrow culture using a standard technique. ${ }^{14}$ In total, 20 GTG banded bone marrow metaphase cells were analyzed. The karyotypes were named according to the International System for Human Cytogenetic Nomenclature. ${ }^{15}$ The cytogenetic analysis performed on 20 metaphase cells identified the presence of a complex, four-way $(4 ; 9 ; 22 ; 21)(\mathrm{q} 24 ; \mathrm{q} 34 ; \mathrm{q} 11 ; \mathrm{q} 22) \mathrm{Ph}$ chromosome translocation (Figure 1) and an additional chromosomal aberration $\mathrm{t}(1 ; 20)(\mathrm{p} 36 ; \mathrm{p} 11)$. In the present case, the Philadelphia translocation is likely to be the first event, followed by the further rearrangement involving chromosomes

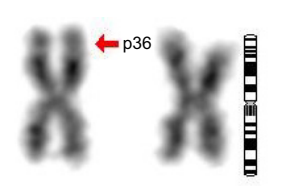

1

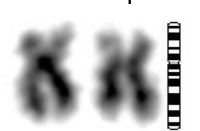

6

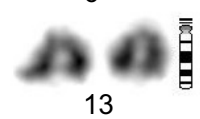

13

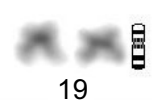

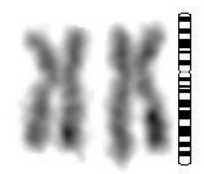

2

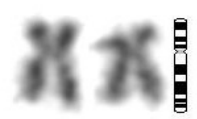

7

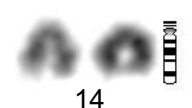

14

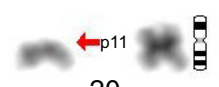

20

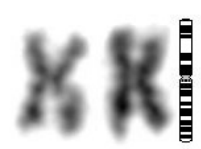

3

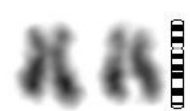

8

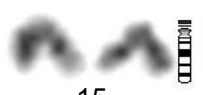

15

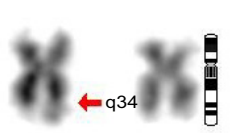

9
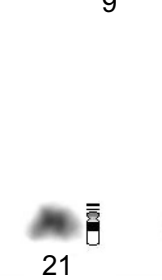
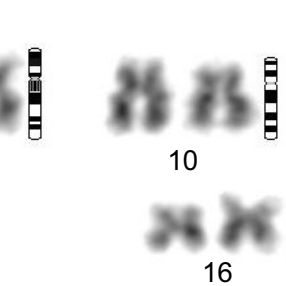

10
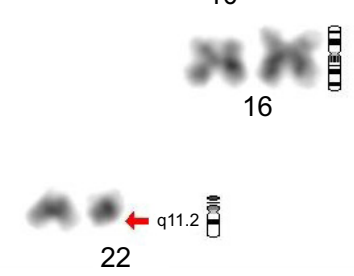

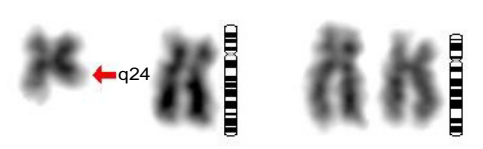

4 5

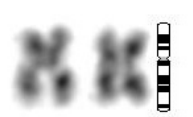

11

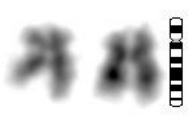

12
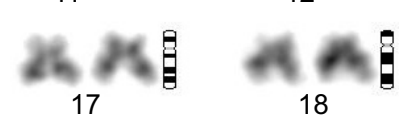

18

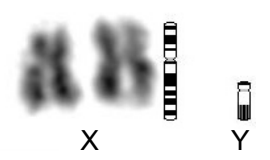

Figure I Cytogenetic analysis.

Notes: Chromosome analysis by $\mathrm{G}$ banding showing a complex, four-way $(4 ; 9 ; 22 ; 21)(q 24 ; q 34 ; q 11 ; q 22)$ Philadelphia chromosome translocation and an additional chromosomal aberration $\mathrm{t}(\mathrm{I} ; 20)(\mathrm{p} 36 ; \mathrm{pl} \mathrm{I})$. Of the two chromosomes $2 \mathrm{I}$, is visible only the normal one; the other is split among 4,9 and 22 chromosomes. Arrows indicate rearranged chromosomes. 
$4 \mathrm{q}$ and $21 \mathrm{q}$, in a step by step sequence. The additional $\mathrm{t}(1 ; 20)$ (p36;p11) can be considered an independent event.

\section{FISH analysis}

FISH analysis using a BCR/ABL dual color dual fusion probe (Figure 2), showed the presence of a variant BCR/ABL translocation in approximately $80 \%$ of interphase nuclei with a single fusion signal; two BCR and two ABL signals showing the splitting of one of the two expected fusion signals, thus suggested the involvement of additional chromosomes and, therefore, we extended the analysis to metaphase spreads. Painting probes for chromosomes 4 and 22 confirmed the presence of a translocation involving chromosomes 4 and 22, generating two derivative chromosomes, one normal chromosome 4 and one normal 22 (Figure 3).

\section{Molecular testing}

At diagnosis, nested RT-PCR was done on peripheral blood and bone marrow showing a b3a2 BCR/ABL fusion gene; at 3 months real-time quantitative RT-PCR was perfomed using TaqMan system and a BCR-ABL1 transcripts' level $<10 \%(0.0829 \%)$ according to the International Scale was found (MMR).

\section{Cytological analysis}

Examination of aspirated bone marrow revealed a slightly hypercellular marrow with granulocytic hyperplasia. Histological examination of the placenta revealed the presence of chorionic villus with characters of development corresponding

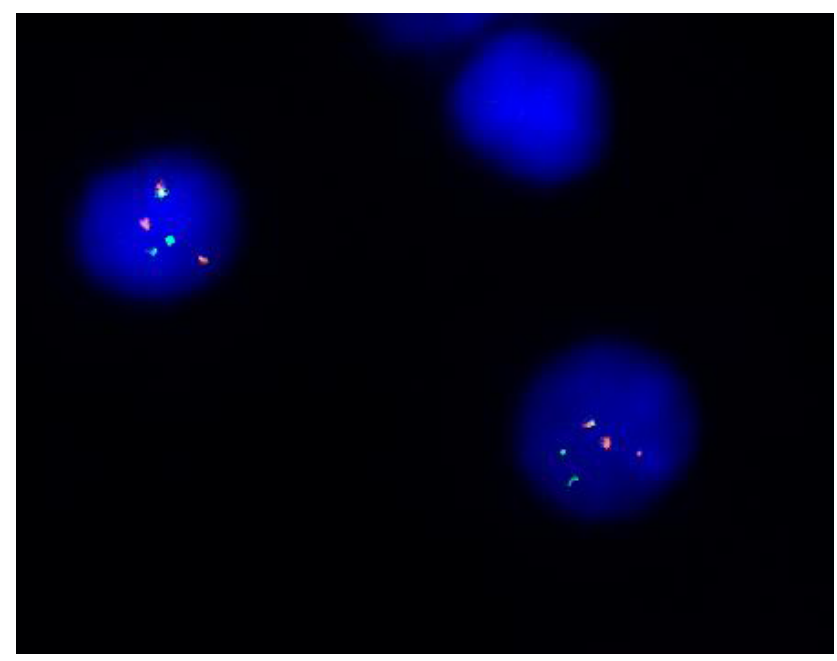

Figure 2 FISH analyses with Bcr and Abl probes.

Note: Interphase nuclei showing one BCR/ABL fusion signal (orange/green spot), a normal Abl signal (orange large spot), a normal Bcr signal (green large spot), and the splitting of the reciprocal Bcr/Abl fusion signal into one small orange and one small green spot due to the further rearrangements of chromosomes 9 and 22. Abbreviation: $\mathrm{FISH}$, fluorescence in situ hybridization.

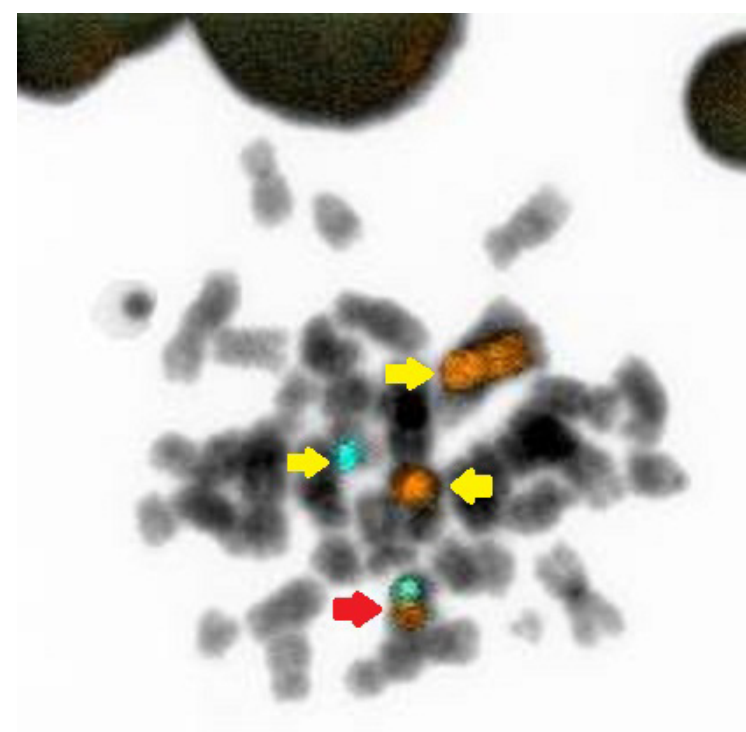

Figure $3 \mathrm{FISH}$ analyses with chromosomes 4 and 22 probes.

Notes: Image showing the results of FISH on a metaphase spread, with painting probes for chromosomes 4 (orange) and 22 (aqua). The yellow arrows indicate normal chromosome 4 (orange - in the upper), residual chromosome 4 (orange - in the middle) and chromosome 22 (aqua); the red arrow indicates derivative chromosome 4 (orange + aqua).

Abbreviation: FISH, fluorescence in situ hybridization.

to gestational age ( 32 weeks), with intervillous space characterized by the presence of increased lympho-monocytic and granulocytic elements. Membranes and umbilical cord were without significant histological alterations.

\section{Discussion}

In this report we describe a unique case of a 36-year-old female with CP-CML diagnosed at 18 weeks of pregnancy, characterized by a new complex variant translocation $\mathrm{t}(4 ; 9 ; 22 ; 21)(\mathrm{q} 24 ; \mathrm{q} 34 ; \mathrm{q} 11 ; \mathrm{q} 22)$ and $\mathrm{t}(1 ; 20)(\mathrm{p} 36 ; \mathrm{p} 11)$ as ACA. The rearrangement was analyzed by cytogenetic and FISH tests. To the best of our knowledge, this translocation has not been observed in CML previously and particularly in a pregnant patient. The management of CML diagnosed during pregnancy is very challenging due to the physiological changes, including those in hematological parameters which accompany the pregnancy, that may mask the symptoms. Previously, it seemed that CML diagnosed during pregnancy was associated with low birth weight and preterm birth, but this is no longer described in more recent reports. Reassuringly, the course of the disease does not appear to be affected by pregnancy. ${ }^{16}$ The prothrombotic potential of a normal pregnancy is well recognized as a result of a physiological increase in hemostatic factors and prothrombotic proteins in addition to the physical obstruction of venous blood flow. As a result, thrombosis continues to be the most common 
cause of maternal morbidity and this may be compounded in the myeloproliferative diseases where there is an associated elevation in the platelet count. Therapeutic approaches for CML diagnosed in pregnancy include supportive care in the form of leukapheresis and platelet pheresis and interferon-a (IFN-a), while the use of tyrosine kinase inhibitors (TKIs) is controversial. ${ }^{17}$ Leukapheresis and platelet pheresis allow avoidance of potentially teratogenic drugs but are not easily available and tolerable. The frequency of these procedures depends on white cell and platelet count. IFN-a, which has a high molecular weight, does not cross the placenta and does not inhibit DNA synthesis; for these reasons it is considered safe in pregnancy. Among TKIs, imatinib is the most studied. It does not cross the placenta but various congenital abnormalities have been described after exposure to imatinib in the first trimester, when placenta formation is not completed, probably due to platelet derived growth factor receptor alpha inhibition. Thus, imatinib and generally all TKIs should be avoided in the first trimester and during organogenesis. Dasatinib crosses the placenta and leads to considerable levels in fetal plasma. It should be avoided in all pregnant patients since it is responsible for fetal hydrops and severe fetal bicytopenia ${ }^{18}$ even if normal pregnancies during exposure have been reported. ${ }^{19-21}$ Nilotinib does not cross the placenta in a significative concentration and does not seem to be teratogenic, but data are limited. A recent review summarized reported cases and provided recommendations of management of pregnancy in CML; so far no case of pregnancy in a CML patient associated with bosutinib and ponatinib therapy has been described. ${ }^{22}$ However, as in our case, treatment is not always mandatory; it is necessary if white cell count exceeds $100 \times 10^{9} / \mathrm{L}$ and platelet count exceeds $500 \times 10^{9} / \mathrm{L}$, as reported by Milojkovic and Apperley. ${ }^{23}$ Low-molecular-weight heparin, as well as aspirin, can be used. Regarding the complex karyotype of our patient, Philadelphia translocation is likely to be the first event, followed by the further rearrangement involving chromosomes $4 \mathrm{q}$ and $21 \mathrm{q}$, in a step by step sequence. The additional $\mathrm{t}(1 ; 20)(\mathrm{p} 36 ; \mathrm{p} 11)$ can be considered an independent event. $\mathrm{t}(1 ; 20)$ has been reported associated with a high rate of recurrent first trimester abortions in a large family; ${ }^{24}$ in our case, the mother did not spontaneously abort her child during the pregnancy despite the presence of this aberration. Mkrtchyan et $\mathrm{al}^{25}$ suggested two possible mechanisms which may be involved in the formation of variant or complex translocations. The first is a single event in which rearrangement due the simultaneous breakage of several chromosomes is by mismatched joining. The second is a multi-step mechanism in which a classical $\mathrm{Ph}$ translocation is followed by further translocation events involving chromosomes 9 and 22, plus a second, third, and subsequent event leading to a multiple-way translocation. Concerning treatment, after strict observation, low dose aspirin and a safe delivery, our patient was treated with dasatinib, a second-generation TKI, at a dosage of $100 \mathrm{mg}$ per day, considering early age and the complex, never described before, translocation of uncertain prognostic significance. Dasatinib is a multi-targeted kinase inhibitor of BCR/ABL, SRK, c-KIT, ephrin receptors, and PDGFRB and from 2012 it can be used also in first line treatment of CML. Treatment was well tolerated with no significant side effects. After 3 months the patient showed a complete cytogenetic response and MMR achieving an optimal response according to ELN recommendations, proving dasatinib's efficacy also in the case of complex karyotype.

\section{Conclusion}

We report a novel case of CP-CML with a new complex variant translocation $\mathrm{t}(4 ; 9 ; 22 ; 21)(\mathrm{q} 24 ; \mathrm{q} 34 ; \mathrm{q} 11 ; \mathrm{q} 22)$ and an ACA t(1;20)(p36;p11). Notably, the patient concerned showed a good tolerability and a good response to dasatinib also, in the presence of a complex karyotype.

\section{Acknowledgment}

Written informed consent was obtained from the patient for publication of this case report and any accompanying images.

\section{Disclosure}

The authors report no conflicts of interest in this work.

\section{References}

1. Rowley JD. A new consistent chromosomal abnormality in chronic myelogenous leukaemia identified by quinacrine fluorescence and Giemsa staining. Nature. 1973;243:290-293.

2. Fabarius A, Leitner A, Hochhaus A, et al. Impact of additional cytogenetic aberrations at diagnosis on prognosis of CML: long-term observation of 1151 patients from the randomized CML Study IV. Blood. 2011;118(26):6760-6768.

3. Baccarani M, Deininger MW, Rosti G, et al. European LeukemiaNet recommendations for the management of chronic myeloid leukemia: 2013. Blood. 2013;122(6):872-884.

4. Fioretos T, Johansson B. Chronic myeloid anchor leukemia. In: Heim S, Mitelman F, editors. Cancer Cytogenetics. Hoboken, NJ: Wiley-Blackwell. 2009:179-207.

5. Huntly BJ, Reid AG, Bench AJ, et al. Deletions of the derivative chromosome 9 occur at the time of the Philadelphia translocation and provide a powerful and independent prognostic indicator in chronic myeloid leukemia. Blood. 2001;98:1732-1738.

6. Quinta's-Cardama A, Cortes J. Molecular biology of bcr-abl1-positive chronic myeloid leukemia. Blood. 2009;113(8):1619-1630.

7. Castagnetti F, Testoni N, Luatti S, et al. Deletions of the derivative chromosome 9 do not influence the response and the outcome of chronic myeloid leukemia in early chronic phase treated with imatinib mesylate: GIMEMA CML Working Party analysis. J Clin Oncol. 2010;28(16): 2748-2754. 
8. Marzocchi G, Castagnetti F, Luatti S, et al. Variant Philadelphia translocations: molecular-cytogenetic characterization and prognostic influence on frontline imatinib therapy, a GIMEMA Working Party on CML analysis. Blood. 2011;117(25):6793-6800.

9. Luatti S, Castagnetti F, Marzocchi G, et al. Additional chromosomal abnormalities in Philadelphia-positive clone: adverse prognostic influence on frontline imatinib therapy: a GIMEMA Working Party on CML analysis. Blood. 2012;120(4):761-767.

10. Deininger MW, Cortes J, Paquette R, et al. The prognosis for patients with chronic myeloid leukemia who have clonal cytogenetic abnormalities in philadelphia chromosome-negative cells. Cancer. 2007; 110(7):1509-1519.

11. Verma D, Kantarjian H, Shan J, et al. Survival outcomes for clonal evolution in chronic myeloid leukemia patients on second generation tyrosine kinase inhibitor therapy. Cancer. 2010;116(11):2673-2681.

12. Lee SE, Choi SY, Bang JH, et al. The long-term clinical implications of clonal chromosomal abnormalities in newly diagnosed chronic phase chronic myeloid leukemia patients treated with imatinib mesylate Cancer Genet. 2012;205(11):563-571.

13. Lichtman MA. Acute myelogenous leukemia. In: Williams Hematology. Vol. 1047, 6th ed. New York, NY: McGraw-Hill; 2001.

14. Shaffer LG, Slovak ML, Cambell LJ, editors. ISCN 2009: An International System for Human Cytogenetic Nomenclature. Basel: Karger. 2009:40-44.

15. Brothman AR, Persons DL, Shaffer LG. Nomenclature evolution: Changes in the ISCN from the 2005 to the 2009 edition. Cytogenet Genome Res. 2009;127(1):1-4.
16. Apperley J. CML in pregnancy and childhood. Best Pract Res Clin Haematol. 2009;22(3):455-474.

17. Milojkovic D, Apperley J. State-of-the-art in the treatment of chronic myeloid leukaemia. Curr Opin Oncol. 2008;20(1):112-121.

18. Cortes JE, Abruzzese E, Chelysheva E, Guha M, Wallis N, Apperley JF The impact of dasatinib on pregnancy outcomes. Am J Hematol. 2015;90(12):1111-1115.

19. Kroll T, Ames MB, Pruett JA, Fenske TS. Successful management of pregnancy occurring in a patient with chronic myeloid leukemia on dasatinib. Leuk Lymphoma. 2010;51(9):1751-1753.

20. Conchon M, Sanabani SS, Serpa M, et al. Successful pregnancy and delivery in a patient with chronic myeloid leukaemia while on dasatinib therapy. Adv Hematol. 2010;2010:136252.

21. Bayraktar S, Morency B, Escalón MP. Successful pregnancy in a patient with chronic myeloid leukaemia exposed to dasatinib during the first trimester. BMJ Case Rep. 2010;2010:pii:bcr0520102975.

22. Abruzzese E, Trawinska MM, de Fabritiis P, Baccarani M. Management of pregnant chronic myeloid leukemia patients. Expert Rev Hematol. 2016;9(8):781-791.

23. Milojkovic D, Apperley JF. How I treat leukemia during pregnancy. Blood. 2014;123(7):974-984.

24. Madan K, Kleinhout J. First trimester abortions associated with a translocation t(1;20)(p36;p11). Hum Genet. 1987;76(1):109.

25. Mkrtchyan H, Ghazaryan S, Avetisyan G, et al. Novel complex t(V;9;22) rearrangements in three cases with chronic myeloid leukemia and a rare translocation in a case with classical Philadelphia chromosome. Oncol Rep. 2008;20(1):99-104.
Therapeutics and Clinical Risk Management

\section{Publish your work in this journal}

Therapeutics and Clinical Risk Management is an international, peerreviewed journal of clinical therapeutics and risk management, focusing on concise rapid reporting of clinical studies in all therapeutic areas, outcomes, safety, and programs for the effective, safe, and sustained use of medicines. This journal is indexed on PubMed Central, CAS,

\section{Dovepress}

EMBase, Scopus and the Elsevier Bibliographic databases. The manuscript management system is completely online and includes a very quick and fair peer-review system, which is all easy to use. Visit http://www.dovepress.com/testimonials.php to read real quotes from published authors.

Submit your manuscript here: http://www.dovepress.com/therapeutics-and-clinical-risk-management-journal 